\title{
Erratum to: On the Measurement of Turbulence Over Complex Mountainous Terrain
}

\author{
Ivana Stiperski $^{1} \cdot{\text { Mathias W. } \text { Rotach }^{1}}^{1}$
}

Published online: 7 December 2015

C) Springer Science+Business Media Dordrecht 2015

\section{Erratum to: Boundary-Layer Meteorol DOI 10.1007/s10546-015-0103-z}

The funding agency information in the Acknowledgements was not included in the original publication of the article. The revised Acknowledgments is provided in this erratum.

"We would like to thank A. Grachev and an anonymous reviewer for valuable comments. Special thanks go to Reto Stauffer, Markus Dabernig and Manuel Presser of the University of Innsbruck for their valuable advice and help in statistical analysis in Sect. 5.We are happy to acknowledge the contributions of students in the analysis of the turbulence data: René Schubert (University of Kiel) initially helped with part of the statistics leading to Fig. 2, and Masters' students of the Alpine Meteorological Field course are thanked for their contribution to field campaign measurements that led to Fig. 5 and Sect. 4. The i-Box infrastructure has been established through funding of the University of Innsbruck. Financial support for this work from the Austrian Science Fund (FWF), under grant \#P 26290-N26 is thankfully acknowledged. All data used herein are available from the authors."

The online version of the original article can be found under doi:10.1007/s10546-015-0103-z.

Ivana Stiperski

ivana.stiperski@uibk.ac.at

1 Institute of Atmospheric and Cryospheric Sciences, University of Innsbruck, Innsbruck, Austria 